\title{
PKS 1117+146: GRAVITATIONAL LENS OR MICRO LOBES
}

\author{
M. BONDI AND M. GARRETT \\ NRAL, Jodrell Bank, UK \\ AND \\ L. GURVITS \\ JIVE and NFRA, Dwingeloo, NL \\ Astro Space Center, Moscow, Russia
}

PKS $1117+146$ is a high power radio source $\left(\mathrm{L}_{327 M H z}=5.4 \times 10^{26} \mathrm{~W} / \mathrm{Hz}\right)$ identified with a galaxy of 20.1 red magnitude at $\mathrm{z}=0.362$ (de Vries et al. 1995). At this redshift 1 mas $\simeq 2.9 \mathrm{pc}\left(\mathrm{H}_{0}=100 \mathrm{~km} / \mathrm{s}^{-1} \mathrm{Mpc}^{-1}\right)$. Based on the properties of the radio spectra, PKS $1117+146$ is classified as a GigaHertz Peaked Spectrum source (GPS) (Stanghellini et al. 1990). The GPS are powerful but physically small (sub-galactic sizes) radio sources with turnovers in their radio spectra at $\nu \sim 1 \mathrm{GHz}$. They are supposed to be isotropically emitting radio sources confined by exceptional dense circumnuclear gas (O'Dea et al. 1991) or still relatively young (Fanti et al. 1990). PKS $1117+146$ is also a low frequency variable (LFV) with no sign of variability at $\nu>1 \mathrm{GHz}$ (Padrielli et al. 1987, Mitchell et al. 1994). The low frequency variability is caused by propagation effects in the interstellar medium of our Galaxy (Mantovani et al. 1990, Spangler et al. 1993). PKS $1117+146$ was observed with VLBI global arrays at $608 \mathrm{MHz}$ (Padrielli et al. 1991), at $327 \mathrm{MHz}$ (Altschuler et al. 1995), and at $1667 \mathrm{MHz}$ (Bondi et al. 1996). All the maps are in agreement showing a compact double structure with components separated by about 70 mas. Flux densities and separation of the two components derived from VLBI and MERLIN (see below) maps are listed in Table 1 . The flux ratios of the two components from the VLBI observations are very similar, and the spectral index is relatively flat $(\alpha \simeq 0.3-0.4)$, even if the strong low frequency variability can introduce uncertainties. The similarity of the VLBI morphology and spectral properties of the two components suggested that $1117+146$ could be a possible gravitational lens candidate prompting for higher frequency observations. We observed PKS $1117+146$ with MERLIN at $22 \mathrm{GHz}$ in 


\begin{tabular}{ccccc}
\hline $\begin{array}{c}\text { Frequency } \\
\text { MHz }\end{array}$ & $\begin{array}{c}\text { Flux Density } \\
\text { SE Comp. }\end{array}$ & $\begin{array}{c}\text { Flux Density } \\
\text { NW Comp. }\end{array}$ & $\begin{array}{c}\text { d } \\
\text { mas }\end{array}$ & $\begin{array}{c}\text { p.a. } \\
\text { degress }\end{array}$ \\
\hline 327 & 2.08 & 1.74 & 70 & -62 \\
608 & 1.66 & 1.32 & 69 & -68 \\
1667 & 1.32 & 1.03 & 78 & -60 \\
23000 & $\sim 0.11$ & $\sim 0.06$ & 86 & -62 \\
\hline
\end{tabular}

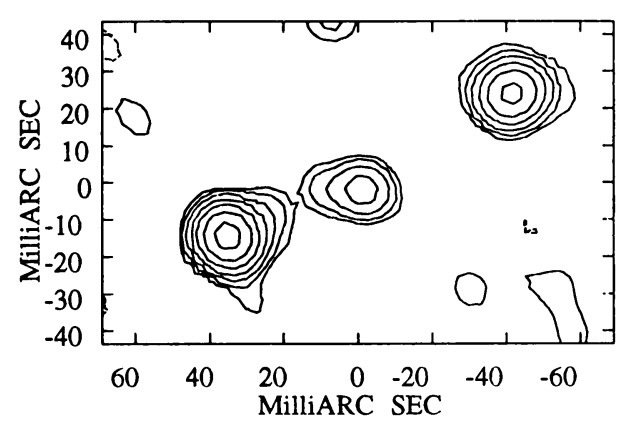

Figure 1. Merlin $22 \mathrm{GHz}$ map

March 1993. MERLIN observations reveal for the first time a weak central component with a total flux density of about $20 \mathrm{mJy}$ (Fig.1). From Table 1 we can note that the P.A. between the components is constant at all the frequencies while the separation between the peak flux densities significantly increases at higher frequencies. This is the expected behaviour if the 2 components are 2 lobes with hot-spot at the outer edges. The MERLIN map at $22 \mathrm{GHz}$ seems to rule out the possibility that the morphology of PKS $1117+146$ is caused by gravitational lensing.

Acknowledgements: MB acknowledges European Union for financial support as an EU Fellow under contract CHBGCT920112.

\section{References}

Altschuler, D., et al., 1995, in press

Bondi, M., et al., 1996, in preparation

de Vries, W.H., Barthel, P.D., Hes, R., 1995, A\&A Suppl. Ser. in press

Fanti, R., et al., 1990, $A \& A$ 231,333

O'Dea, C.P., Baum, S., Stanghellini, C., 1991, ApJ 380, 660

Padrielli, L., et al., 1987, $A \& A$ Suppl. Ser. 67, 63

Padrielli, L., et al., 1991, $A \& A$ 249, 351

Mantovani, F., et al., 1990, $A \& A$ 233, 535

Mitchell, K.J., et al., 1994, ApJ Suppl. Ser. 93, 441

Spangler, S.R., et al., 1993, $A \& A$ 267, 213

Stanghellini, C., et al., 1990, $A \& A$ 233, 379 